\title{
New research on pandemic influenza at the World Congress of Epidemiology, Edinburgh, 7-11 August 2011
}

J A Summers ${ }^{1}$, N Wilson'1, M G Baker (michael.baker@otago.ac.nz) ${ }^{1}$

1. Department of Public Health, University of Otago, Wellington, New Zealand

This brief report outlines selected highlights of new research on pandemic influenza that was presented at the 2011 World Congress of Epidemiology. This event was held in Edinburgh, United Kingdom between 7 and 11 August. The conference had over 1,400 attendees from an estimated 65 countries. The theme was Changing populations, changing diseases: epidemiology for tomorrow's world and was facilitated by the International Epidemiological Association. Abstracts of the conference posters and slide presentations have been published [1].

While the content of the conference was dominated by non-communicable diseases, we focus here on pandemic influenza given the large amount of new research generated by the 2009 pandemic. Additionally, we review research investigating other areas related to the field of influenza.

\section{Epidemiology}

Various studies explored the impact that age can play during an influenza pandemic. Azambuja's study (P1-7), investigated the possible long-term effect of influenza exposure on the risk of developing chronic disease (such as coronary heart disease later in life) in agespecific cohorts exposed to various influenza $A$ subtypes. A more comprehensive study by this author [2] suggests that residual influenza $\mathrm{A}\left(\mathrm{H}_{1} \mathrm{~N}_{1}\right)$ antibodies in cohorts aged 60 years and older helped to protect against the severe effects of the $2009 \mathrm{H}_{1} \mathrm{~N}_{1}$ influenza pandemic. Two further studies explored the impact of age, but focused on its role in mortality risk during an influenza pandemic: Lee et al. (P1-213) found that influenza $A\left(\mathrm{H}_{1} \mathrm{~N}_{1}\right)_{2009}$-related mortality amongst children in Malaysia was highest in the under two year-olds. In contrast, age-specific mortality risks during the $\mathrm{H}_{1} \mathrm{~N}_{1}$ 1918 influenza pandemic in Iceland and New Zealand were notably increased amongst young adults ( $\mathrm{P}_{1}$ 524). Whilst neither of these findings is particularly surprising given previous research, they do reiterate the role that host factors play in mortality risk during an influenza pandemic.
Analysis of influenza epidemiology in different populations was another area of research: One study explored the incidence of influenza $\mathrm{A}\left(\mathrm{H}_{1} \mathrm{~N}_{1}\right)_{2009}$ amongst pregnant women in a region of Scotland, using questionnaires, blood samples and clinical outcomes (P2-428). The provisional results reported few cases of infection with no evidence for a relationship between pregnancy and increased rates of hospital admission, and no relationship between rates of admission and several potential risk factors. The former finding contrasts with the conclusion of a systematic review which did find that pregnancy was a risk factor for hospital admission and death from pandemic influenza [3]. Another study (P2429) investigated the incidence of seropositivity for influenza $A\left(\mathrm{H}_{1} \mathrm{~N}_{1}\right)_{2009}$ both before and during the winter peak in Scotland amongst selected healthcare workers (HCWs). Seropositivity was found to be only $10.3 \%$ in the middle of the pandemic; therefore the majority of the sample was still susceptible to infection after the peak. The provisional results found no difference in seropositivity between frontline and non-frontline HCWs. Ethnicity was found to be an important factor in determining mortality risk from pandemic influenza in New Zealand (P2-489). The study suggests that Pacific peoples and indigenous Māori in New Zealand suffered disproportionate levels of mortality during two influenza pandemics of the 2oth century as well as the $\mathrm{H}_{1} \mathrm{~N}_{1}$ 2009 pandemic, although the size of the disparities appears to have declined with progressive pandemics.

\section{Transmission routes and control measures}

The relationship between weather variability and transmission routes for influenza infection was considered by $\mathrm{Hu}$ et al. (P2-418). Using national data collections for a city in Australia, they found that the number of weekly influenza cases increased (with a lag of one week) as temperature decreased and/or rainfall increased. Two studies explored influenza transmission in confined spaces: Ishola et al. (P1-180) investigated the effect of mass gatherings on influenza transmission by reviewing previous research using a restricted search strategy. It was concluded that some restrictions on mass gatherings in particular situations 
may result in reduced transmission, but more research is needed to feed into policy frameworks as the association is not consistent. The second study explored transmission amongst passengers on a long-haul flight who were exposed to symptomatic cases of influenza $\mathrm{A}\left(\mathrm{H}_{1} \mathrm{~N}_{1}\right) 2009$ infection ( $\mathrm{P}_{1-388)}$. It concluded that close proximity to symptomatic cases resulted in a small, but measurable, risk of infection during such flights. A further study in the airport setting assessed the use of a self-reported symptom questionnaire and temperature measurement of all symptomatic and randomly selected asymptomatic travellers (P1-295). It was concluded that due to the low prevalence of influenza infection and low sensitivity of the screening, border screening in the event of an influenza pandemic may not be effective in identifying all, or even the majority, of infected travellers.

Three studies (SP3-49, $P_{1-323}$, and $P_{1-363)}$ found evidence for a beneficial effect of interrupting the transmission route of $\mathrm{H}_{1} \mathrm{~N}_{1} 2009$ pandemic influenza amongst school children in Japan by antiviral drugs and/or school closure (of varying length and timing). However, Uda et al. ( $\mathrm{P}_{1-363)}$ suggested that the positive effect of herd immunity in the school environment may have been adversely affected by early school closure, with lower cumulative incidence rates in schools that delayed closure until a later date. Using data on the impact of the pandemic on Japanese schools, modelling work suggested that cumulative incidence rates would be likely to vary in the range of $23-44 \%$, depending on the size of the school community ( $\left.\mathrm{P}_{1-77}\right)$. The wide range of cumulative incidence rates amongst individual schools was suggested to be the result of the random nature of infectious disease transmission; however other potential variables (environmental and socio-economic factors) are being examined.

Whilst not directed specifically at influenza control, a few studies investigated hygiene practises in developing countries (SP6-60, P2-529 and P1-305). A study by Onyeonoro et al. of the poor hygiene practises of HCWs in a Nigerian hospital highlighted the need for providing better hygiene facilities in developing countries (SP6-60). Although this study did not link to infectious disease outcomes in the hospital, there is little doubt that poor hygiene is associated with a number of detrimental outcomes. For example, a household survey in Pakistan found poor hygiene and sanitation was directly associated with delayed development and/or underweight/stunting in children irrespective of socioeconomic and geographic variables (P2-529).

\section{Prevention}

Of the few studies which investigated influenza prevention, most explored seasonal and pandemic vaccine efficacy and uptake in particular subpopulations, e.g. individuals with compromised immune systems:

Evidence was presented for a booster effect of a second dose of the pandemic influenza $A\left(\mathrm{H}_{1} \mathrm{~N}_{1}\right) 2009$ monovalent vaccine in haemodialysis patients (P2-485) and haemato-oncology patients (P2-420). Nevertheless, the latter authors did suggest that the use of rituximab (a cancer treatment) within one year of vaccination may counter vaccine efficacy.

A presentation by Jacobsen et al. ( $\left.\mathrm{P}_{1-183}\right)$ focused on seasonal influenza vaccine amongst cancer patients. It evaluated the levels of seasonal influenza vaccination in California before and after the diagnosis of prostate cancer and found that vaccination levels increased after diagnosis, suggesting that preventive care for influenza was successfully provided to this more vulnerable group.

Uptake of influenza vaccine in a selected sample of elderly people in Brazil (SP6-5) was found not to be affected by socio-economic variables; however, national target levels of uptake were only achieved amongst people with diabetes.

\section{Surveillance and modelling}

The assessment of surveillance methods is an important field of study after the first wave of a new influenza pandemic. When investigating the prevalence of potential influenza $A\left(\mathrm{H}_{1} \mathrm{~N}_{1}\right)_{2009}$ cases through selfreporting via a telephone survey after the end of the pandemic in Brazil, Souza et al. (SP3-92) concluded that telephone surveillance during a pandemic may be beneficial in providing timely information. The estimated prevalence of pandemic influenza cases was found to be greater amongst females, younger adults, and those with higher education. In neighbouring Argentina, Figar et al. ( $\left.\mathrm{P}_{1-423}\right)$ suggested that a private healthcare information system in Buenos Aires captured more timely information during the 2009 pandemic than the system in the public health sector. These authors therefore advocated for more integration of data capture. Data from the private healthcare information system indicated significantly higher mortality in 2009 compared with previous years for both influenza-like illness (ILI) and severe acute respiratory infection (SARI). Elsewhere in South America, Horch et al. (SP3-87) studied the sentinel surveillance (implemented in response to the 2009 pandemic) of SARI and ILI in selected hospitals in Paraguay. They found evidence of increased data collection and also improved characterisation of respiratory viruses. Additionally, ILI cases occurred more frequently amongst young adults, whilst SARI cases occurred mainly in children under five years and adults over 60 years. A further study based in Paraguay documented the substantial burden of influenza ( $\left.\mathrm{P}_{1-105}\right)$ by evaluating swabs of individuals exhibiting SARI symptoms for influenza subtypes (influenza $A\left(\mathrm{H}_{3} \mathrm{~N}_{2}\right), A\left(\mathrm{H}_{1} \mathrm{~N}_{1}\right)$ and $\left.B\right)$. Of the samples tested, $18 \%$ were positive for influenza, suggesting a substantial SARI burden from this infectious agent.

A study of the Spanish public health system during the 2009 pandemic identified the problem of low compliance by health workers with a protocol for limiting diagnostic testing to only those with serious illness 
(P1-147). Also, modelling of infectious diseases morbidity (including influenza) in Japan was found to be useful in forecasting temporal patterns, therefore potentially contributing to future disease risk management plans ( $\mathrm{P}_{1-177)}$.

\section{Conclusion}

Influenza pandemics tend to occur several times per century and can cause extreme mortality (notably the 1918/19 pandemic). Seasonal influenza also accounts for a substantial burden of illness worldwide. Therefore, new research in the wake of the 2009 influenza pandemic is commendable and should continue to be promoted by health research funders and health service providers themselves. In particular, such research may help countries reflect on the 2009 pandemic experience [4], and then revise their national pandemic plans and upgrade their influenza-related surveillance systems. The high costs associated with developing pandemic vaccines and storing antiviral drugs also highlights the need for new research on the optimal use of these pharmaceuticals - especially given the financial constraints on public health systems around the world.

\section{Acknowledgments}

The lead author's attendance at the conference was funded by the following sources: the Wellington Medical Research Foundation Incorporated (New Zealand), the Postgraduate Research Student Support Fund (University of Otago, Wellington, New Zealand), and the Division of Health Sciences PhD Travel Fund (University of Otago, Wellington, New Zealand).

\section{References}

1. Programme and abstracts. International Epidemiological Association (IEA) World Congress of Epidemiology; 7-11 August 2011; Edinburgh, Scotland. J Epidemiol Community Health. 2011;65(Suppl 1):1-473.

2. Azambuja MIR. Influenza Recycling and Secular Trends in Mortality and Natality. British Actuarial Journal. 2009;15(Supp):123-50.

3. Mosby LG, Rasmussen SA, Jamieson DJ. 2009 Pandemic influenza $A\left(\mathrm{H}_{1} \mathrm{~N}_{1}\right)$ in pregnancy: a systematic review of the literature. Am J Obstet Gynecol. 2011 Feb 21. Epub ahead of print.

4. Leung GM, Nicoll $A$. Reflections on pandemic $\left(\mathrm{H}_{1} \mathrm{~N}_{1}\right)$ 2009 and the international response. PLoS Med. 2010;7(10):pii:e1000346. 